\title{
Development of an In Planta system to monitor phosphorus status by agroinfiltration and agroinjection
}

\author{
Jia-Ying Lin • Tzyy-Jen Chiou • Hsiao-Feng Lo • \\ Shu-I Lin 10
}

Received: 24 November 2015 / Accepted: 9 June 2016/Published online: 28 June 2016

(C) Springer International Publishing Switzerland 2016

\begin{abstract}
Background and Aims An optimal supply of phosphate (Pi) fertilizer is important to ensure crop yield and quality and to maintain agricultural sustainability. In this study, an in planta monitoring system was developed to conveniently monitor the $\mathrm{P}$ status of crops.

Methods A phosphate starvation-induced gene (TPSI1) from tomato (Solanum lycopersicum L.) was used to reflect $\mathrm{P}$ status in plants. Using agroinfiltration and agroinjection techniques, the GUS reporter gene driven by the TPSI1 promoter was transiently expressed in tobacco (Nicotiana benthamiana) leaves and tomato fruits to reflect their P status.

Results We showed that the vegetative growth of tobacco and the yield and quality of tomato were affected by the supply of Pi fertilizer. We further demonstrated that

Responsible Editor: Philip John White.

Electronic supplementary material The online version of this article (doi:10.1007/s11104-016-2959-1) contains supplementary material, which is available to authorized users.

J.-Y. Lin · H.-F. Lo · S.-I. Lin $(\bowtie)$

Department of Horticulture and Landscape Architecture, National Taiwan University, No. 1, Sec. 4, Roosevelt Rd, Taipei 10617, Taiwan

e-mail: shuilin@ntu.edu.tw

T.-J. Chiou

Agricultural Biotechnology Research Center, Academia Sinica, No. 128, Sec. 2, Academia Rd, Taipei 11529, Taiwan
\end{abstract}

the expression of a GUS reporter gene driven by the TPSI1 promoter could accurately report on $\mathrm{P}$ status in tobacco leaves and tomato fruits in real time. Expression of the GUS reporter gene was independent of deficiencies in mineral nutrients other than $\mathrm{P}$, demonstrating the specificity of this system.

Conclusions The above results indicate that the employed agroinfiltration/agroinjection-based diagnostic system is useful for monitoring $\mathrm{P}$ status in plants.

Keywords Phosphorus - Tobacco · Tomato ·

Agroinfiltration · Agroinjection

\section{Introduction}

An optimal supply of Pi fertilizer is important to ensure crop productivity and to maintain agricultural $\mathrm{P}$ sustainability

Phosphorus $(\mathrm{P})$ plays important roles in plant growth and development. $P$ is one of the least available nutrients in soil (Raghothama 1999; Raghothama and Karthikeyan 2005). Most $\mathrm{P}$ in soil is unavailable for plants due to its precipitation, adsorption or conversion to organic forms (Schachtman et al. 1998). Inorganic phosphate (Pi), the major form of $\mathrm{P}$ that is available for plants, is often at a much lower concentration in soil than in plant tissue (Schachtman et al. 1998; Raghothama 1999). This low P 
availability may limit the growth of plants in natural ecosystems. Therefore, $\mathrm{P}$ availability is important for crop productivity.

To ensure crop productivity, farmers often apply excessive amounts of Pi fertilizer. However, unabsorbed Pi fertilizer may enter aquatic systems and cause the eutrophication of lakes and streams (Holford 1997; Raghothama 1999; Withers et al. 2001; Vance et al. 2003). Pi fertilizer is extracted from Pi rock, which is not renewable and may be exhausted within 50125 years (Raghothama 1999; Vance et al. 2003; Hammond and White 2008; Cordell et al. 2009; Gilbert 2009). A shortage of high-quality Pi rock would increase financial burdens on farmers. Today's food system relies heavily on mined Pi rock to maintain high agricultural productivity; a shortage of Pi rock may also threaten global food security (Cordell and White 2014). In addition to breeding high Pi-use efficiency (PUE) crops, the optimized application of Pi fertilizer to fields is another solution for the sustainable use of $\mathrm{P}$ (Gaxiola et al. 2011).

Tomato is a low Pi-use efficiency plant, due to its low root/shoot ratio and low influx rate (Raghothama 1999). Pi starvation in tomato reduces the Pi concentration in the roots and shoots, the roots and shoots dry weights, stomatal conductance, net $\mathrm{CO}_{2}$ assimilation, and fruit and stem diameters, while increasing the root/shoot biomass ratio (Clarkson and Scattergood 1982; Biddinger et al. 1998; Fujita et al. 2003). Therefore, an adequate supply of $\mathrm{Pi}$ is necessary to ensure yield and quality in the commercial production of tomato.

Development of a smart plant for monitoring the $\mathrm{P}$ status in planta

The optimal application of Pi fertilizer requires an accurate reporting system to evaluate the need for Pi fertilizers. By the time that Pi starvation is diagnosed by visual systems, such as anthocyanin accumulation, it is often too late to avoid reductions in yield and/or quality (Hammond and White 2008; Hammond et al. 2011). Chemical analyses of P concentrations in soil and/or plant materials by colorimetric assays or inductively coupled plasma (ICP) spectroscopic techniques are the most common approaches used to evaluate Pi fertilizer needs in the field (Murphy and Riley 1962; Pittman et al. 2005; Hammond and White 2008; Adesanwo et al. 2013). However, because of the inaccessibility of most soil P (Holford 1997), measurement of soil P may not correctly reflect the availability of Pi or the true status of the plants. Moreover, $\mathrm{P}$ elemental analysis is timeconsuming and requires advanced equipment. Improved techniques that are more convenient for quickly measuring the $\mathrm{P}$ status of plants are desirable.

Plants sense and respond to $\mathrm{P}$ deficiency by altering gene expression levels before visible signs of $\mathrm{P}$ deficiency develop (Hammond and White 2008). Hammond et al. developed a smart plant technology by transforming Arabidopsis with a construct containing a Pi starvation-inducible promoter coupled to a visible marker (Hammond et al. 2003; Hammond and White 2008). The visible marker can report on whether plants are encountering Pi starvation stress. AtSQD1 is induced when Arabidopsis encounters Pi deficiency and functions in sulfolipid biosynthesis (Essigmann et al. 1998). Transgenic Arabidopsis expressing a $\beta$-glucuronidase (GUS) reporter gene driven by the promoter of AtSQD1 was used to monitor P status in this plant. GUS activity increased when plants encountered Pi deficiency and was reduced when Pi was resupplied (Hammond et al. 2003; Hammond et al. 2004; Hammond and White 2008). This transgenic plant was termed a smart plant. Similarly, Kamiya et al. created a transgenic plant expressing the magnesium-responsive gene calcium/proton exchanger 3 (CAX3) fused to a luciferase (LUC) reporter gene to monitor magnesium concentration (Kamiya et al. 2012).

Applications of an agroinfiltration/agroinjection transient expression system

Agroinfiltration is a transient expression system in which suspensions of Agrobacterium tumefaciens expressing the desired transgene constructs are infiltrated into leaves (Schöb et al. 1997). Compared to stable transgenic plants, agroinfiltration is a rapid and simple method to perform functional analyses of genes without expensive instruments (Yang et al. 2000). Agroinfiltration has been successfully used in promoter analyses (Yang et al. 2000), and this system has been used to demonstrate that promoters of the pathogen-responsive genes $P R 1 a$ and $P R 2 d$ from tobacco are responsive to salicylic acid (SA) and tobacco mosaic virus (TMV) infection (Yang et al. 2000). The responsiveness of the promoter of the heat shock gene Gm17.5 from soybean to heat treatment was also demonstrated using an agroinfiltration system (Yang et al. 2000). In addition, agroinjection was developed to express transgenes in fruit by injecting $A$. tumefaciens suspensions 
harboring constructs of interest (Orzaez et al. 2006). This method has been successfully used to demonstrate the responsiveness of the promoter of the heat shockregulated gene $H S P 70$ to heat treatment in tomato fruit (Orzaez et al. 2006).

Use of an agroinfiltration/agroinjection-based diagnostic system for monitoring $\mathrm{P}$ status

Although the smart plant idea was proposed in 2003 (Hammond et al. 2003), only Arabidopsis has been used as a model system. The requirement of transgenic plant generation may hamper the application of this system. Because the promoter activity in response to external stresses can be estimated in live plants via a combination of agroinfiltration/agroinjection and biotic or abiotic treatments (Yang et al. 2000; Orzaez et al. 2006), we used this system with a construct containing the promoter of a gene induced by Pi starvation and a visible marker to prove the concept of an "agroinfiltration/ agroinjection-based diagnostic system" to monitor $\mathrm{P}$ status in planta.

The expression level of the tomato phosphate starvation-induced gene (TPSI1) under Pi-sufficient conditions is extremely low but can be rapidly and highly induced in the roots and leaves of tomato in response to Pi starvation (Liu et al. 1997). TPSI1 expression is repressed when $\mathrm{Pi}$ is resupplied to Pi-starved plants (Liu et al. 1997). TPSI1 is specifically regulated by $\mathrm{Pi}$ and not by other nutrients, such as nitrogen $(\mathrm{N})$, potassium $(\mathrm{K})$, iron $(\mathrm{Fe})$, calcium $(\mathrm{Ca})$ or magnesium (Mg) (Liu et al. 1997; Biddinger et al. 1998). Therefore, the rapid, highly induced, reversible and specific response of TPSI1 suggests that it can be used to quickly and accurately reflect $\mathrm{P}$ status in tomato. Furthermore, TPSI1 homologs exist in many plant species (Lin et al. 2009; Chiou and Lin 2011), including Arabidopsis thaliana (Burleigh and Harrison 1999; Martín et al. 2000; Shin et al. 2006), Medicago truncatula (Burleigh and Harrison 1997, 1998, 1999) and Oryza sativa (Wasaki et al. 2003; Hou et al. 2005), and it functions via similar regulatory mechanisms, making TPSI1 a good candidate for this system because of its potential application to other plant species. As a proofof-concept for such a monitoring system, in this study we combined an agroinfiltration/agroinjection transient expression system with the $\mathrm{P}_{T P S I 1}:: G U S$ construct to report on the $\mathrm{Pi}$ status in tobacco leaves and tomato fruits.

\section{Materials and methods}

Plant materials and growth conditions

Seeds were surface-sterilized using $1 \%$ sodium hypochlorite $(\mathrm{NaOCl})$ with $0.5-1 \mu$ Tween 20 . Surfacesterilized seeds of tobacco (Nicotiana benthamiana) and 'Micro-Tom' tomato (Solanum lycopersicum L.) were grown in hydroponic culture with half-strength modified nutrient solution (Hoagland and Arnon 1950; Millner and Kitt 1992) with $500 \mu \mathrm{M} \mathrm{KH}_{2} \mathrm{PO}_{4}$. A subset of the tobacco seedlings was grown under Pi-sufficient conditions $\left(500 \mu \mathrm{M} \mathrm{KH} \mathrm{KH}_{2} \mathrm{PO}_{4}\right)$ for 20 days (+Pi0d). Roots and leaves were collected separately for Pi assays and for root and shoot fresh weight (F.W.) analyses. A subset of $+\mathrm{PiOd}$ seedlings were transferred to $\mathrm{Pi}$ sufficient (+Pi, $\left.500 \mu \mathrm{M} \mathrm{KH}_{2} \mathrm{PO}_{4}\right)$ or Pi-deficient (-Pi, $0 \mu \mathrm{M} \mathrm{KH}_{2} \mathrm{PO}_{4}$ ) nutrient solutions for 1 day (+Pi1D and $-\mathrm{Pi} 1 \mathrm{D}), 3$ days (+Pi3D and -Pi3D), 4 days (+Pi4D and Pi4D), 5 days (+Pi5D and -Pi5D), 6 days (+Pi6D and Pi6D), 7 days (+Pi7D and -Pi7D) or 8 days (+Pi8D and -Pi8D). For the Pi-resupply experiments, -Pi3D seedlings were transferred to Pi-sufficient solution for 1 day (-Pi3DRe1D), 2 days (-Pi3DRe2D), 3 days (-Pi3DRe3D), 4 days (-Pi3DRe4D) or 5 days (-Pi3DRe5D). The above materials were also collected for Pi assays and F.W. analyses. For the different nutrient deficiency treatments, 20-day-old tobacco seedlings grown in half-strength modified nutrient solution with $500 \mu \mathrm{M} \mathrm{KH} \mathrm{KH}_{2} \mathrm{PO}_{4}$ were maintained in the original full nutrient solution or transferred to a solution in which specific nutrient elements were removed for 7 days. To compensate, an equivalent concentration of ions was supplied when specific ions were omitted. The details were as follows: for $-\mathrm{N}, \mathrm{Ca}\left(\mathrm{NO}_{3}\right)_{2}$ and $\mathrm{KNO}_{3}$ were replaced with $\mathrm{CaCl}_{2}$ and $\mathrm{KCl}$; for $-\mathrm{P}, \mathrm{KH}_{2} \mathrm{PO}_{4}$ was replaced with $\mathrm{KCl}$; for $-\mathrm{K}, \mathrm{KNO}_{3}$ and $\mathrm{KH}_{2} \mathrm{PO}_{4}$ were replaced with $\mathrm{Ca}\left(\mathrm{NO}_{3}\right)_{2}$ and $\mathrm{NaH}_{2} \mathrm{PO}_{4}$; for $-\mathrm{Ca}$, $\mathrm{Ca}\left(\mathrm{NO}_{3}\right)_{2}$ was replaced with $\mathrm{NH}_{4} \mathrm{NO}_{3}$; for $-\mathrm{Mg}$, $\mathrm{MgSO}_{4}$ was replaced with $\mathrm{CaSO}_{4}$; for $-\mathrm{S}, \mathrm{MgSO}_{4}$ was replaced with $\mathrm{MgCl}_{2}$; and for -Fe, NaFeEDTA was omitted from the nutrient solution. Leaves and roots were collected separately for the Pi assay. The nutrient solution was replenished every 5 days. Tobacco was grown in a phytotron at the National Taiwan University (NTU) with $25 / 20{ }^{\circ} \mathrm{C}$ day/night temperatures and 16-h light/8-h dark cycles with light being supplied by high pressure sodium lamps (HPS) at an intensity of $120 \mu \mathrm{mol} \mathrm{m}^{-2} \mathrm{~s}^{-1}$. 
For tomato fruit assays, 145-day-old 'Micro-Tom' seedlings grown under Pi-sufficient $\left(+\mathrm{Pi}, 500 \mu \mathrm{M} \mathrm{KH_{2 }} \mathrm{PO}_{4}\right)$ conditions were transferred to Pi-sufficient $(+\mathrm{Pi}, 500 \mu \mathrm{M}$ $\left.\mathrm{KH}_{2} \mathrm{PO}_{4}\right)$ and Pi-deficient (-Pi, $0 \mu \mathrm{M} \mathrm{KH} \mathrm{KH}_{2} \mathrm{PO}_{4}$ ) nutrient solutions for 5 days (+Pi5D and -Pi5D), 10 days (+Pi10D and -Pi10D), 15 days (+Pi15D and -Pi15D) or 20 days (+Pi20D and -Pi20D). For Pi-resupply experiments, -Pi10D seedlings were transferred to Pi-sufficient solution for 5 days (-Pi10DRe5D) or 10 days (-Pi10DRe10D). The F.W., Pi concentration, total fruit weight and total soluble solid content (TSS) of these materials were determined. The nutrient solution was replenished every 5 days. Tomatoes were grown in a greenhouse at the NTU at temperatures between 16 and $24^{\circ} \mathrm{C}$ and photosynthetically active radiation supplied at $158-445 \mu \mathrm{mol} \mathrm{m}{ }^{-2} \mathrm{~s}^{-1}$.

Transgene constructs and Agrobacterium transformation

The pCambia 1301 vector, which contains the cauliflower mosaic virus (CaMV) 35S promoter fused with a GUS reporter, was used as the $\mathrm{P}_{35 S}: G U S$ positive control. To construct the $\mathrm{P}_{T P S I}:: G U S$ construct, the TPSI1 promoter was first amplified by PCR using the primers 5'-gaacaacacttggcgaaataca-3' and 5'ccctaaaagttggtggcaaa-3'. The generated PCR product was $1561 \mathrm{bp}$ in length. The PCR product was cloned into the pGEM-T Easy Vector (Promega, Madison, USA) and confirmed by sequencing. The TPSI1 promoter was then subcloned into pMDC164 (Curtis and Grossniklaus 2003), which harbored a GUS reporter, using Gateway recombination technology (Invitrogen, USA). To build the $\mathrm{P}_{355}:$ :LUC construct, the LUC gene was excised from the pJD301 vector using NcoI and SacI restriction enzymes. The $\mathrm{SacI}$ restriction site was then filled in to form a blunt end. The vector pCambia 1302 was digested with $N c o I$ and Pml1 and ligated with the above LUC insert to obtain the $\mathrm{P}_{355}:$ :LUC construct.

Plasmid DNA was isolated using the Plasmid DNA Extraction System (Viogene, Taiwan) following the manufacturer's instructions. Plasmid DNA was then transformed into A. tumefaciens strain C58C1 as described in a previous study (Hofgen and Willmitzer 1988) with minor modifications. To prepare competent cells, A. tumefaciens $\mathrm{C} 58 \mathrm{C} 1$ was cultured in $5 \mathrm{~mL}$ of YEP medium $\left(10 \mathrm{~g} \mathrm{~L}^{-1}\right.$ Bacto-peptone, $10 \mathrm{~g} \mathrm{~L}^{-1}$ yeast extract, and $5 \mathrm{~g} \mathrm{~L}^{-1} \mathrm{NaCl}$ ) with shaking at $28{ }^{\circ} \mathrm{C}$ for $16 \mathrm{~h}$. The bacteria were subcultured in $100 \mathrm{~mL}$ of YEP medium and grown until $\mathrm{OD}_{550}=0.5 \sim 0.8$. After the bacteria were centrifuged at
$5000 \mathrm{rpm}$ for $10 \mathrm{~min}$ at $4{ }^{\circ} \mathrm{C}$, the pellet was washed with TE buffer (10 mM Tris and $1 \mathrm{mM}$ EDTA) and resuspended in $10 \mathrm{~mL}$ of YEP. Then, $500-\mu \mathrm{L}$ aliquots of bacteria were quickly frozen in liquid nitrogen and stored at $-80{ }^{\circ} \mathrm{C}$. For transformation, the competent cells were first thawed on ice. Next, $10 \mu \mathrm{L}$ of plasmid DNA was added to the thawed competent cells, which were incubated on ice for $5 \mathrm{~min}$, in liquid nitrogen for $5 \mathrm{~min}$ and at $37^{\circ} \mathrm{C}$ for $5 \mathrm{~min}$. After the addition of $1 \mathrm{~mL}$ of YEP medium, the cells were incubated at $28^{\circ} \mathrm{C}$ with shaking for $2 \sim 4 \mathrm{~h}$. The bacteria were plated on YEP plates containing appropriate antibiotics and incubated for 2 days at $28{ }^{\circ} \mathrm{C}$. A single confirmed colony was picked for the following experiments.

Agroinfiltration of tobacco leaves

Agroinfiltration was performed in parts of the tobacco plants three days before the end of Pi treatment $(+\mathrm{Pi},-\mathrm{Pi}$ and Pi resupply) (Fig. 1a) and nutrient-deficient treatment (Fig. 1b). Agroinfiltration was performed following a previously described method (Bendahmane et al. 2000; Liu et al. 2010; Liu et al. 2012) with minor modifications. Briefly, suspensions of Agrobacterium C58C1 containing the above constructs were cultured in YEP medium with appropriate antibiotics with shaking at $28{ }^{\circ} \mathrm{C}$ for $16 \mathrm{~h}$. The bacteria were then subcultured in YEP medium containing appropriate antibiotics, $10 \mathrm{mM}$ MES and $20 \mu \mathrm{M}$ acetosyringone (AS) with shaking for 5-7 h, until the $\mathrm{OD}_{600}$ of the cultures reached approximately 1 . After the cultures were centrifuged at $5000 \mathrm{rpm}$ at $4{ }^{\circ} \mathrm{C}$ for $10 \mathrm{~min}$, the cell pellets were resuspended in infiltration medium $[10 \mathrm{mM} \mathrm{MgCl}, 10 \mathrm{mM}$ MES ( $\mathrm{pH}=5.6)$, and $200 \mu \mathrm{M}$ $\mathrm{AS}]$ to $\mathrm{OD}_{600}=1.0$. The cell suspension was stored in the dark at room temperature for $3 \mathrm{~h}$ and then used to infiltrate near fully expanded leaves using a needleless syringe. For normalization, equal volumes of Agrobacterium suspensions carrying the $\mathrm{P}_{355}: L U C$ construct were mixed with Agrobacterium suspensions carrying the $\mathrm{P}_{T P S I 1}:: G U S$ or $\mathrm{P}_{355}:$ GUS constructs for co-infiltration. Three days after agroinfiltration, the leaves were used for GUS staining or frozen in liquid nitrogen for GUS and LUC activity assays.

Agroinjection of tomato fruits

Agroinjection was performed in parts of the tomato plants three days before the end of $\mathrm{Pi}$ treatment $(\mathrm{Pi},-\mathrm{Pi}$ and $\mathrm{Pi}$ resupply) (Fig. 1c). Agroinjection was performed following a previously described method (Orzaez et al. 2006) 
(a)

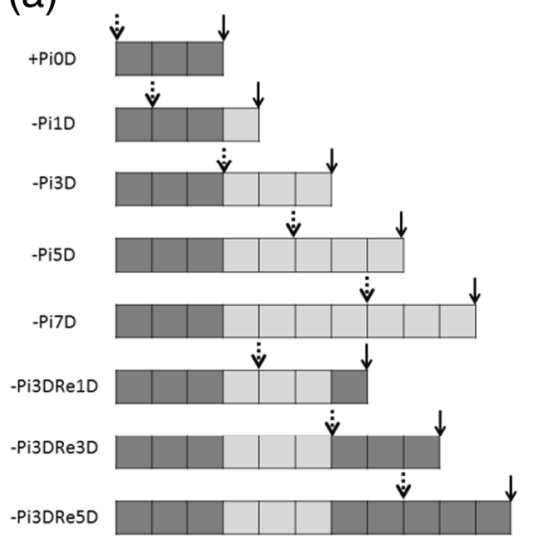

Fig. 1 Time frames of agroinfiltration in tobacco leaves and agroinjection in tomato fruits. a Tobacco plants were grown under Pi-sufficient (+Pi) conditions or subjected to Pi-deficient $(-\mathrm{Pi})$ and Pi-resupply $(\mathrm{Re})$ treatments of different durations. Agroinfiltration was performed three days before the assays (GUS staining and GUS and LUC activity assays). b For different nutrient deficiency treatments, tobacco plants were grown in nutrient solutions in which a single specific nutrient element was absent (Deficient)

with minor modifications. A C58C1 Agrobacterium suspension was prepared following the above method for agroinfiltration. Green-stage tomato fruits were injected with the Agrobacterium suspension using a syringe with a needle. The needle was introduced into the fruit via the stylar apex, and the Agrobacterium suspension harboring the constructs was gently injected into the fruit. For normalization, fruits were co-injected with equal volumes of an Agrobacterium suspension carrying the $\mathrm{P}_{355}:$ LUC construct and Agrobacterium suspensions carrying $\mathrm{P}_{T P S I I}:: G U S$ or $\mathrm{P}_{355}:: G U S$ constructs. Three days after agroinjection, the fruits were used for GUS staining or frozen in liquid nitrogen for GUS and LUC activity assays.

Pi concentration analysis

Pi concentrations were determined as previously described (Ames 1966; Chiou et al. 2006).

Total soluble solids (TSS) content analysis

Red stage tomato fruits were freshly ground with a mortar and pestle, and the TSS content was measured (b)

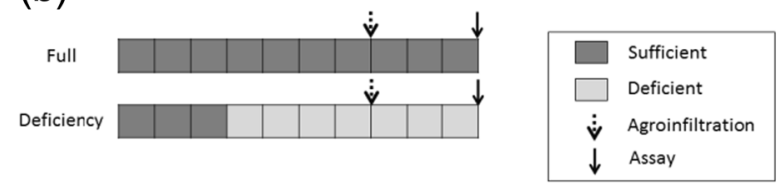

(c)

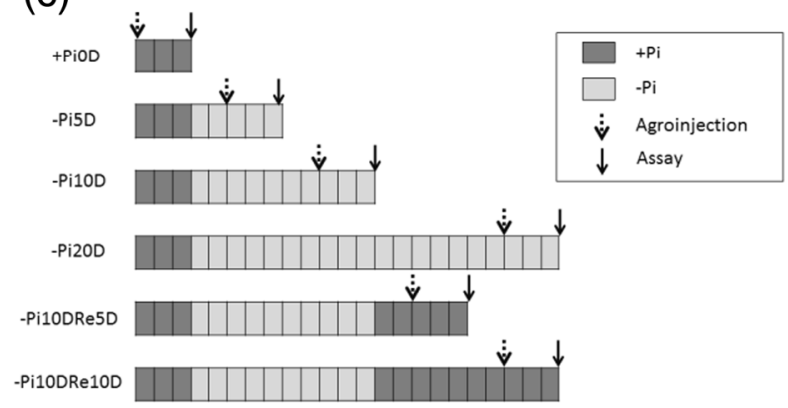

for 7 days. The control plants were maintained in half-strength modified Hoagland nutrient solution with full nutrients (Sufficient). Agroinfiltration was performed three days before the assays (Pi assay, GUS staining, and GUS and LUC activity assays). c Tomato plants were grown under Pi-sufficient $(+\mathrm{Pi})$ conditions or subjected to Pi-deficient ( $-\mathrm{Pi}$ ) and Pi-resupply (Re) treatments of different durations. Agroinjection was performed three days before the assays (GUS staining and GUS and LUC activity assays)

using a Pocket Refractometer PAL-1 (Atago, Tokyo, Japan) following the manufacturer's instructions.

\section{GUS staining and GUS and LUC activity assays}

Because the GUS intensity may change depending on the number of days post-infiltration (dpi) (Wroblewski et al. 2005), all of the materials for GUS staining and GUS and LUC activity assays were collected at 3 dpi. GUS staining was performed as described previously (Jefferson et al. 1987) with minor modifications. The modified GUS staining buffer contained $0.15 \% \mathrm{X}$-gluc (5-bromo-4-chloro-3-indolyl- $\beta$-D-glucuronic acid), $100 \mathrm{mM}$ phosphate buffer ( $\mathrm{pH}=7.0), 10 \mathrm{mM}$ EDTA, $0.5 \mathrm{mM} \mathrm{K}_{3} \mathrm{Fe}(\mathrm{CN})_{6}, 0.5 \mathrm{mM} \mathrm{K}_{4} \mathrm{Fe}(\mathrm{CN})_{6}, 0.1 \%$ Triton $\mathrm{X}-100$ and $10 \%$ methanol. After 3 days of agroinfiltration, a leaf that was approximately $1 \mathrm{~cm} \times 1 \mathrm{~cm}$ was collected from the infiltration site and submerged in GUS staining solution. The samples were vacuum infiltrated for $1 \mathrm{~min}$ under $90 \mathrm{kpa}$ and incubated at $37^{\circ} \mathrm{C}$ for $3 \mathrm{~h}$. After 3 days of agroinjection, the fruits were sectioned at a thickness of $3 \mathrm{~mm}$ and submerged in GUS staining solution. The samples were vacuum infiltrated 
for 3 min under $90 \mathrm{kpa}$, and the tissues were incubated at $37{ }^{\circ} \mathrm{C}$ for $13 \mathrm{~h}$. After the leaf and fruit samples were stained for GUS, the chlorophyll was cleared from the samples for 3 days in $75 \%$ ethanol. Images were acquired using a digital camera (Canon, Tokyo, Japan).

Frozen tissues from tobacco leaves and tomato fruits were ground with a mortar and pestle in liquid nitrogen. The cells were lysed in pre-cooled modified CCRL buffer [0.1 M potassium phosphate buffer $(\mathrm{pH}=7.8)$, $1 \mathrm{mM}$ EDTA, $10 \%$ glycerol, $7 \mathrm{mM} \beta$-mercaptoethanol, $0.1 \%$ Triton X-100 and $2 \%$ polyvinylpolypyrrolidone]. The samples were centrifuged twice at 10,000 rpm for 15 min at $4{ }^{\circ} \mathrm{C}$. The supernatants were used for protein quantification and for GUS and LUC activity assays. The protein contents of the supernatants were quantified as described previously (Bradford 1976) using a protein assay dye reagent (Bio-Rad, USA). Briefly, $4 \mu \mathrm{l}$ of supernatant was mixed with $156 \mu$ l of deionized water and $40 \mu \mathrm{l}$ of protein assay dye reagent (Bio-Rad, USA). Bovine serum albumin (BSA) was used to generate a standard curve. After a 30 -min incubation at $37^{\circ} \mathrm{C}$, the protein concentration was measured using a microplate reader (Infinite 200, Tecan, Switzerland) at $\mathrm{OD}_{595}$. GUS activity was determined as previously described (Jefferson et al. 1987; Spolaore et al. 2001) with minor modifications. Aliquots of the above supernatants were incubated with $10 \mathrm{mM}$ 4-methylumbelliferyl- $\beta$-Dglucuronide (MUG) at $37^{\circ} \mathrm{C}$ for 0,10 or $20 \mathrm{~min}$. The reactions were terminated with $0.2 \mathrm{M} \mathrm{Na}_{2} \mathrm{CO}_{3}$. Released 7-hydroxy-4-methylcoumarin (4-MU) was measured using a microplate reader (Infinite 200, Tecan, Switzerland) with an excitation wavelength of $355 \mathrm{~nm}$ and an emission wavelength of $460 \mathrm{~nm}$. GUS activity was expressed in pmol 4-MU released $\mathrm{min}^{-1} \mu \mathrm{g}^{-1}$ protein. Aliquots of the above supernatants were also used to determine LUC activity using a LUC assay system kit (Promega, Madison, USA) following the manufacturer's instructions. The relative LUC activity was measured using a microplate reader (Infinite 200, Tecan, Switzerland). The relative activity was calculated as the GUS activity divided by the LUC activity of the same tissue that was co-transformed with two constructs containing GUS and LUC reporters.

Experimental design and statistical analysis

Complete randomized design (CRD) was used in this study, and each treatment included 3 replicates. Data were subjected to analysis of variance (ANOVA) using CoStat 6.2 software (CoHort Software, Monterey, CA, USA), and the differences among treatments were determined by Fisher's protected least significant difference (LSD) test at $p<0.05$ levels. All graphs were plotted with SigmaPlot 10.0 (Systat Software Inc., Richmond, CA, USA), and the error bars represent the standard deviation (SD).

\section{Results}

Pi deficiency affects Pi concentrations and growth in tobacco

When tobacco plants were grown under Pi-sufficient conditions, the F.W. of the shoots and roots gradually increased as the growing period increased (Fig. 2a and b). Under Pi-deficient conditions, root F.W. progressively increased as the growing period increased and was comparable to that of plants grown under Pi-sufficient conditions (Fig. 2b). However, Pi deficiency dramatically reduced shoot growth (Fig. 2a). The reduction in shoot F.W. was not observed until four days of starvation treatment (-Pi4D) and the plant growth ceased after six days of treatment (-Pi6D) (Fig. 2a). When Pi was resupplied to $-\mathrm{Pi}$ D plants, the plants quickly resumed growth and the F.W. was able to reach to the level of Pi-replete plants 2 days after resupply (Fig. 2a). This suggests that Pi resupplying following 3-day Pi deficiency can fully recover the plant growth.

Measurement of the Pi concentration revealed that the $\mathrm{Pi}$ concentrations in both roots and leaves remained constant over the growing period when plants were grown under Pi-sufficient conditions (Fig. 2c and d). However, the Pi concentrations in both the roots and leaves were dramatically reduced after one day of Pi-deficient treatment and were reduced further when the Pi-deficient treatment continued. When Pi was resupplied to $-\mathrm{P} 3 \mathrm{D}$ plants for 1 day (-Pi3DRe1D), Pi quickly accumulated in both roots and leaves. The $\mathrm{Pi}$ concentration in these plants was more than threefold greater than that in plants maintained under Pi-sufficient conditions (+Pi4D). The Pi concentration then gradually decreased over time. 
(a)

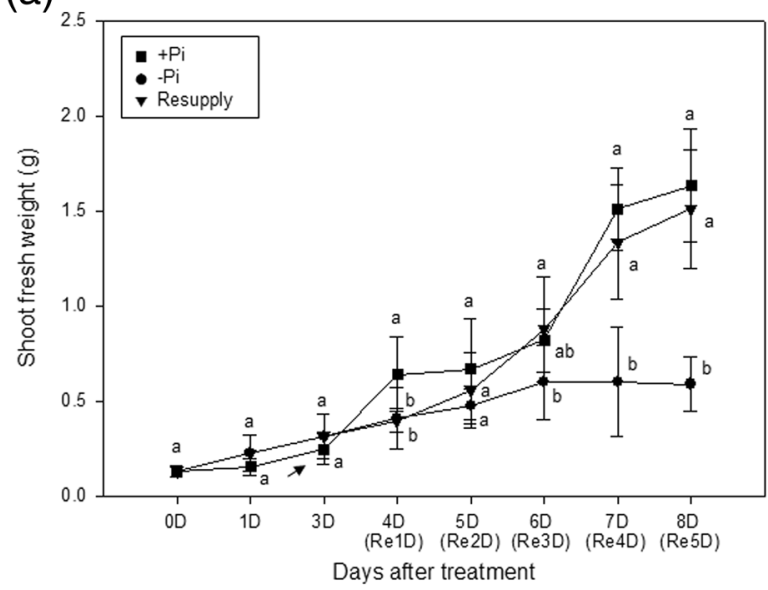

(c)

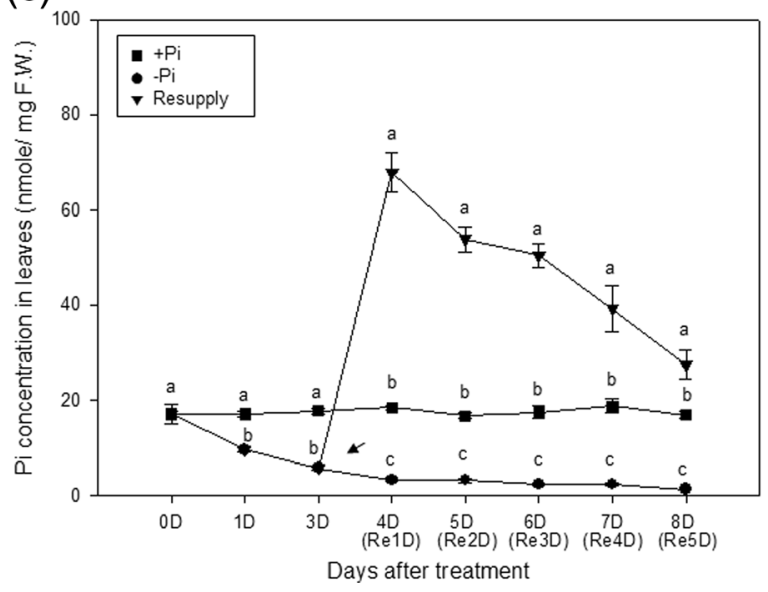

Fig. 2 Effects of Pi treatments for different durations on fresh weight and $\mathrm{Pi}$ concentration in tobacco. Tobacco plants were grown under $\mathrm{Pi}$-sufficient $(+\mathrm{Pi})$ conditions or subjected to $\mathrm{Pi}-$ deficient (-Pi) and Pi-resupply (Re) treatments of different durations. The shoot fresh weight (a), root fresh weight (b), leaf Pi concentration (c) and root Pi concentration (d) were determined.

Specific reporting of the Pi status of tobacco using the agroinfiltration-based diagnostic system

To test whether the constructs in the agroinfiltration system were correctly expressed in the leaves of tobacco grown in a hydroponic culture system, we first performed infiltrations with an Agrobacterium suspension harboring the $\mathrm{P}_{355}:$ GUS construct. Tobacco leaves infiltrated with a suspension of the wild-type Agrobacterium strain C58C1, which did not harbor any constructs, was used as a negative control. GUS

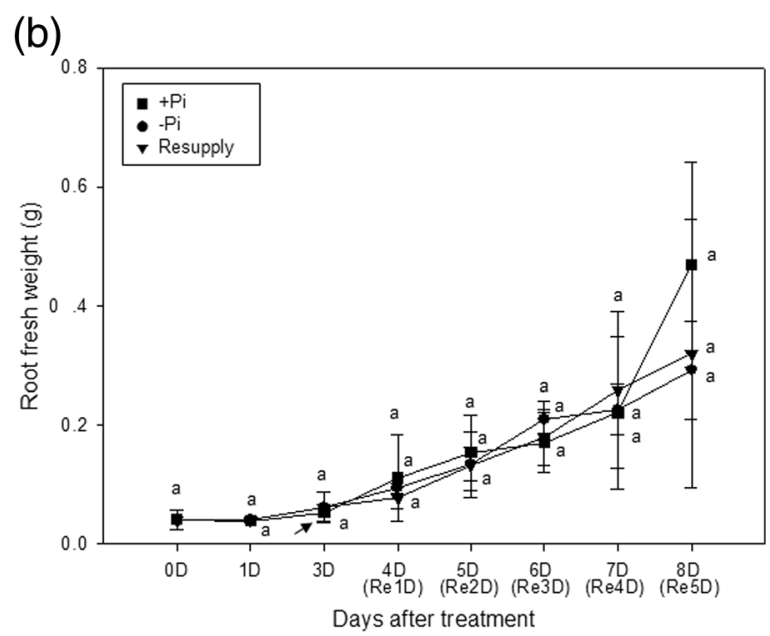

(d)

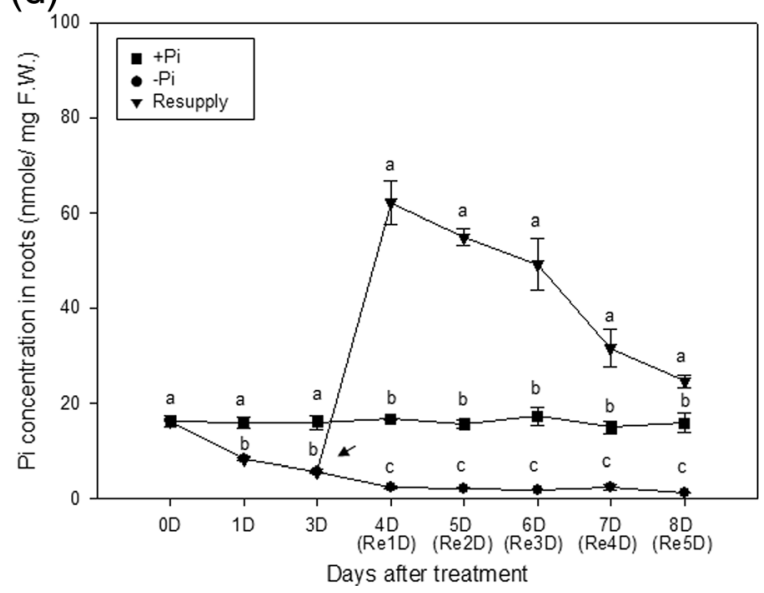

Pi-resupply treatment was initiated after three days of Pi deficiency (arrow). Different letters at each point denote significant differences between Pi treatments at $p<0.05$ using Fisher's protected LSD test. Error bars represent SD of the means of three replicates $(n=3)$

staining was performed 3 days after agroinfiltration. Strong GUS activity was observed in leaves transformed with the $\mathrm{P}_{355}:$ GUS construct, whereas no GUS activity was observed in leaves infiltrated with the wildtype Agrobacterium strain C58C1 (Fig. 3a). When tobacco plants grown under Pi-sufficient, Pi-deficient or Pi-resupply conditions were infiltrated with an Agrobacterium suspension harboring the $\mathrm{P}_{T P S I}:: G U S$ construct, stronger GUS activity was observed in Pi1D plants than in plants grown under Pi-sufficient conditions (+Pi0D) (Fig. 3a). A further increase in 
(a)

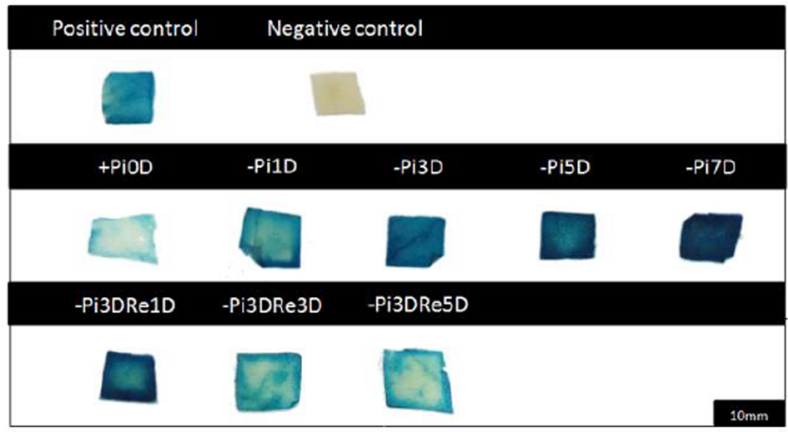

Fig. 3 Effects of Pi treatments for different durations on GUS staining levels and relative GUS activity levels in tobacco leaves after three days of agroinfiltration. a Tobacco plants were grown under Pi-sufficient $(+\mathrm{Pi})$ conditions or subjected to Pi-deficient $(-\mathrm{Pi})$ and Pi-resupply $(\mathrm{Re})$ treatments for different durations. Leaves were used for agroinfiltration and GUS staining. The positive control was transformed with the $\mathrm{P}_{355}:$ :GUS construct. The negative control was not transformed with any construct. Other than the positive and negative controls, all of the leaves used for GUS staining were transformed with the $\mathrm{P}_{T P S I I}:$ GUS construct via agroinfiltration. b Tobacco plants were grown under Pi-sufficient (+Pi) conditions or subjected to Pi-deficient (-Pi) and

GUS activity was observed in tobacco when the duration of Pi-deficient conditions increased. A gradual decline in GUS activity was observed in -Pi3D plants as the number of days of Pi resupply increased.

GUS activity was visually observed via GUS staining and also quantified via a GUS activity assay. To avoid the infiltration of unequal volumes of Agrobacterium suspensions in different leaves, an Agrobacterium suspension harboring the $\mathrm{P}_{355}:$ :LUC construct was co-infiltrated with the $\mathrm{P}_{355}:$ GUS positive or $\mathrm{P}_{T P S I 1}:: G U S$ target constructs and used for normalization. As observed in the GUS staining results, the relative GUS activity driven by the TPSII promoter increased when the duration of the Pi-deficient treatment increased (Fig. 3b). GUS activity was reduced when the number of days of Pi-resupply treatment was increased (Fig. 3b). These results reflect trends opposite from those of the leaf $\mathrm{Pi}$ concentrations (Fig. 2c). After Pi was resupplied to Pi3D plants for 3 days (-Pi3DRe3D), the relative GUS activity was similar to that of plants grown under Pisufficient conditions (+Pi0D) (Fig. 3b). As expected, high relative GUS activity was detected in the positive control (b)

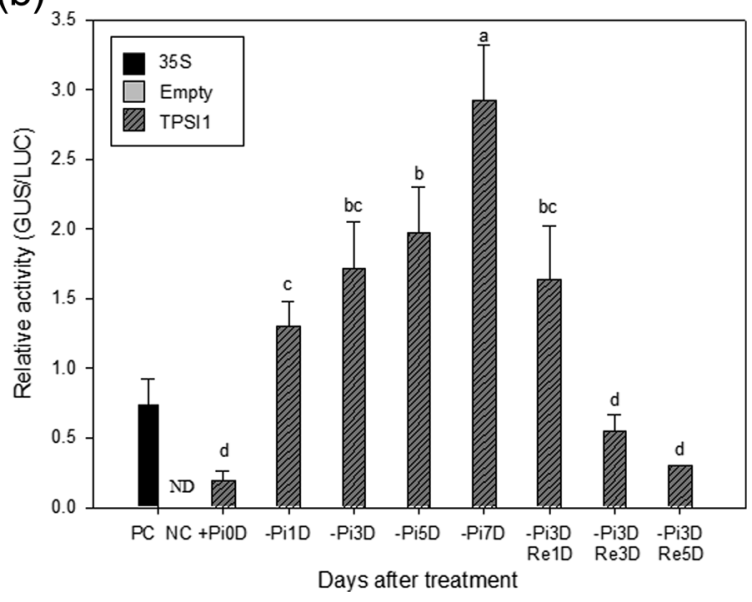

Pi-resupply $(\mathrm{Re})$ treatments for different durations. Leaves were used for agroinfiltration and GUS activity assays. Leaves used for relative GUS-activity assays were transformed with $\mathrm{P}_{355}:$ :GUS (35S, black bars, $\mathrm{PC}$ ), the $\mathrm{P}_{\text {TPSII }}:: G U S$ construct (TPSI1, slashed bars) or no construct (Empty, gray bars, NC) via agroinfiltration. The level of relative GUS activity was normalized to the level of LUC luminescence expressed by the $\mathrm{P}_{355}:: L U C$ construct, which was co-transformed with the $\mathrm{P}_{T P S I}:: G U S$ or $\mathrm{P}_{355}:: G U S$ constructs. Means within each panel followed by the same letters are not significantly different between Pi treatments at $p<0.05$ using Fisher's protected LSD test. Error bars represent the SD values of the means of three replicates $(n=3)$

while the GUS activity in the negative control was undetectable (ND) (Fig. 3b).

To determine the specificity of this system, tobacco plants were cultured under different nutrient solutions in which specific nutrients were absent for seven days. To demonstrate the effectiveness of the imposed nutrient deficiencies, we measured shoot mineral element concentrations after the plants were deprived of different nutrients. As expected, among all treatments, plants had the lowest concentration of each specific mineral element in their shoots when that nutrient was removed from the nutrient solution (Table S1). Before agroinfiltration, we also examined the Pi concentration. Only plants grown under Pideficient conditions had significantly lower Pi concentrations in the leaves (Fig. 4a) and roots (Fig. 4b) compared to plants grown in full nutrient solution. When agroinfiltration was performed, expression of $\mathrm{P}_{35 S}: G U S$ construct resulted in higher GUS activity than the negative control (Fig. $4 \mathrm{c}$ and d). The GUS activity derived from $\mathrm{P}_{T P S I}:: G U S$ was only highly expressed in the plants grown under Pi-deficient conditions (Fig. 4c and d). GUS activity in the leaves of 
(a)

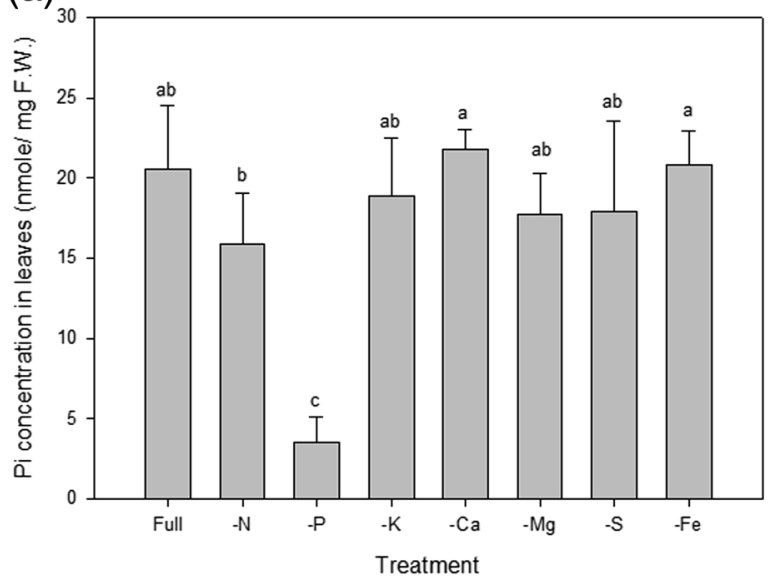

(c)

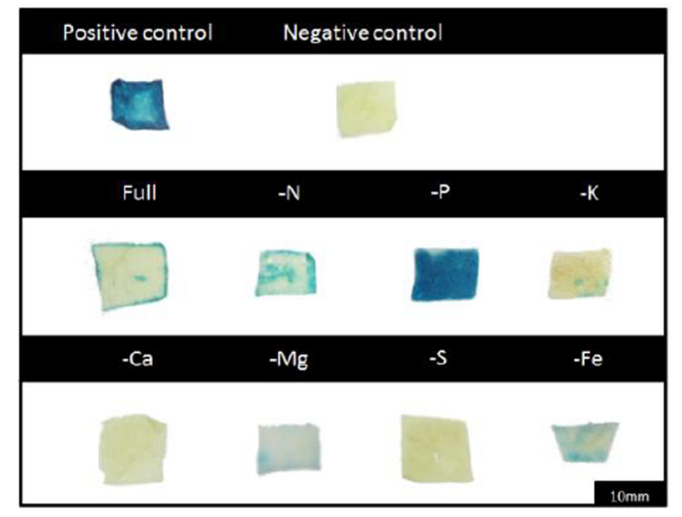

Fig. 4 Effects of different nutrient deficiencies on Pi concentration and GUS activity in tobacco. a Effects of different nutrient deficiencies on leaf Pi concentration in tobacco. For the different nutrient deficiency treatments, the indicated specific nutrient element was withdrawn from the nutrient solution for 7 days. Control (Full) plants were maintained in half-strength modified Hoagland nutrient solution with full nutrients. Means within each panel followed by the same letters are not significantly different between nutrient treatments at $p<0.05$ using Fisher's protected LSD test. Error bars represent the SD values of the means of three replicates $(n=3)$. $\mathbf{b}$ Effects of different nutrient deficiencies on root Pi concentration in tobacco. For the different nutrient deficiency treatments, the indicated specific nutrient element was withdrawn from the nutrient solution for 7 days. Control (Full) plants were maintained in half-strength modified Hoagland nutrient solution with full nutrients. Means within each panel followed by the same letters are not significantly different between nutrient treatments at $p<0.05$ using Fisher's protected LSD test. Error bars represent the SD values of the means of three replicates $(n=3)$. $\mathbf{c}$ Tobacco plants were grown in solutions in which specific nutrient elements were

plants deficient in $\mathrm{N}, \mathrm{K}, \mathrm{Ca}, \mathrm{Mg}, \mathrm{S}$ or $\mathrm{Fe}$ was as low as to that of the plants grown in full nutrient solution (Fig. (b)

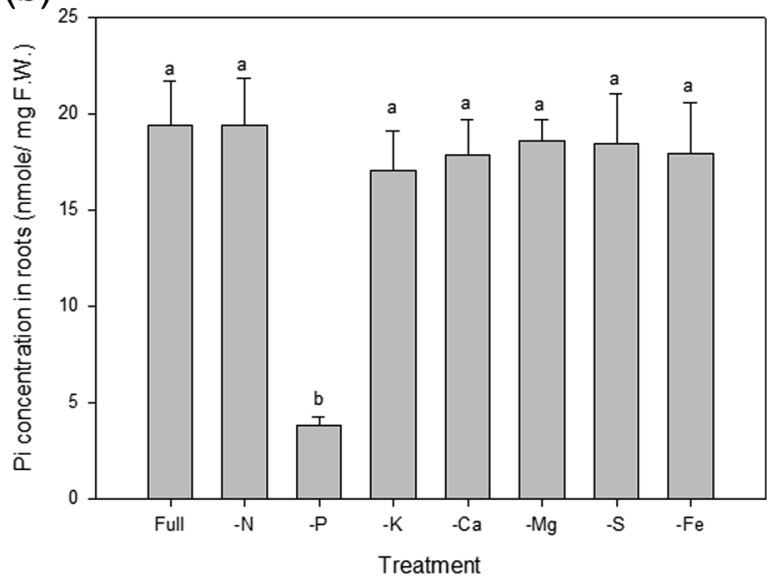

(d)

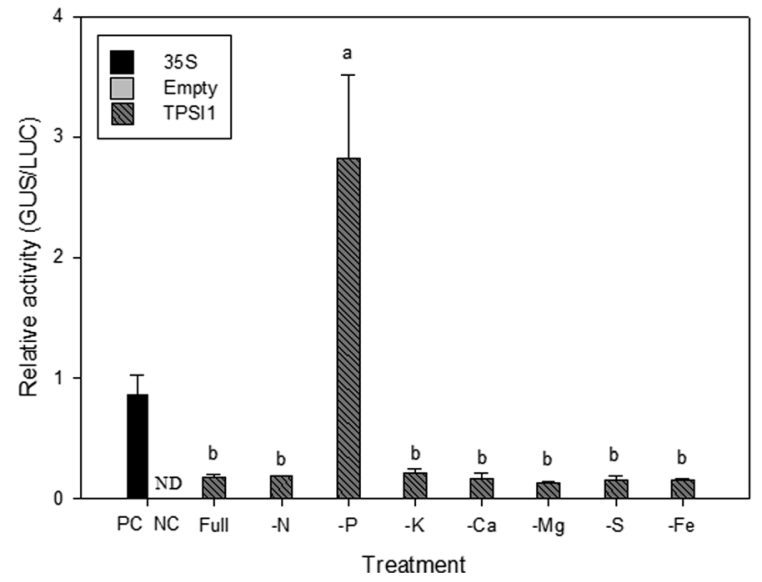

withdrawn for 7 days. Leaves were transformed via agroinfiltration and GUS stained. Other than the positive and negative controls, all of the leaves used for GUS staining were transformed with the $\mathrm{P}_{\text {TPSII }}:$ GUS construct via agroinfiltration. The positive control was transformed with the $\mathrm{P}_{355}: G U S$ construct. The negative control was not transformed with any construct. $\mathbf{d}$ Tobacco plants were grown in solutions in which specific nutrient elements were withdrawn for 7 days. Leaves were transformed via agroinfiltration, and GUS activity was assayed. Leaves used for relative GUS-activity assays were transformed with the $\mathrm{P}_{355}:$ GUS construct (35S, black bars, $\mathrm{PC}$ ), the $\mathrm{P}_{\text {TPSII }}:$ GUS construct (TPSI1, slashed bars) or no construct (Empty, gray bars, NC). The level of relative GUS activity was normalized to the level of LUC luminescence expressed by the $\mathrm{P}_{355}: L U C$ construct, which was co-transformed with the $\mathrm{P}_{T P S I I}:: G U S$ or $\mathrm{P}_{355}:: G U S$ constructs. Means within each panel followed by the same letters are not significantly different between nutrient treatments at $p<0.05$ using Fisher's protected LSD test. Error bars represent the SD values of the means of three replicates $(n=3)$

$4 \mathrm{c}$ and d). These results indicate that this system can specifically monitor the Pi status in plants. 
Pi deficiency affects Pi concentrations and growth in tomato

We studied the effects of Pi starvation on tomato. Tomato plants were grown under Pi-sufficient conditions to the reproductive stage and were then transferred to Pi-sufficient or Pi-deficient nutrient solutions. A subset of plants grown under -Pi conditions for 10 days were then resupplied with $+\mathrm{Pi}$ nutrient solution. No significant difference was observed between the shoot F.W. of plants grown under Pi-sufficient and Pi-deficient conditions for 15 days (+Pi15D and Pi15D) (Fig. 5a). However, the shoot F.W. of Pideficient plants was lower than that of Pi-sufficient plants after 20 days of Pi treatment (+Pi20D and Pi20D) (Fig. 5a).

When the Pi concentrations were examined, plant grown under Pi-deficient conditions for 5 days (-Pi5D) had significantly lower Pi concentrations in fruits than plants grown under Pi-sufficient conditions (+Pi5D) (Fig. 5b). The fruit Pi concentration was reduced further when the Pi-deficient treatment continued. When Pi was resupplied to -Pi10D plants for 5 days (-Pi10DRe5D), the fruit Pi concentration increased quickly, achieving concentrations even higher than those in plants grown under Pi-sufficient conditions (+Pi15D). The fruit Pi concentration subsequently decreased and eventually became comparable to that in plants grown under Pi-sufficient conditions (+Pi20D and-Pi10DRe10D).

The total fruit weight and TSS content at the beginning of the experiment were not significantly different between Pi-sufficient and Pi-deficient plants (Fig. 5c and d). However, the total fruit weight and TSS content were significantly lower in Pi-deficient plants than in Pisufficient plants after 20 days and 15 days of Pi deprivation, respectively (Fig. $5 \mathrm{c}$ and d). Tomato plants subjected to Pi-deficient conditions for 10 days and then resupplied with Pi eventually recovered their shoot F.W., fruit weight and TSS contents to levels similar to those of plants grown continuously under Pi-sufficient conditions (Fig. 5a, c and d).

An agroinjection-based diagnostic system is able to monitor the Pi status in tomato

A transient expression system using agroinfiltration with the $\mathrm{P}_{T P S I}:: G U S$ construct successfully reflected the Pi status in tobacco plants. We also investigated whether the same system could be applied in fruits. Tomato fruits from plants grown under Pi-sufficient conditions were able to successfully express GUS when the agroinjection of a $\mathrm{P}_{355}:$ :GUS construct was performed in green-stage fruits (Fig. 6a and b). As a negative control, the agroinjection of fruits was performed using a suspension of the wild-type Agrobacterium strain $\mathrm{C} 58 \mathrm{C} 1$, which does not harbor any constructs, and the tissues did not display any GUS activity, as determined by GUS staining and GUS activity assays (Fig. 6a and b). After an Agrobacterium suspension harboring the $\mathrm{P}_{T P S I 1}:: G U S$ construct was injected into the fruits, we observed higher GUS activity in the fruits of plants grown under Pi-deficient conditions for 5 days $(-\mathrm{Pi5D})$ than in plants grown under Pi-sufficient conditions (+Pi0D) (Fig. 6a). Stronger GUS activity was observed in plants grown under Pi-deficient conditions for longer periods (Fig. 6a and b). When Pi was resupplied to $-\mathrm{Pi} 10 \mathrm{D}$ plants for 5 or 10 days (-Pi10DRe5D or -Pi10DRe10D), the GUS activity was reduced to a level similar to that in plants grown under Pi-sufficient conditions (+Pi0D) (Fig. 6a and b).

Although TPSII is specifically regulated by $\mathrm{Pi}$ and not by other nutrients (Liu et al. 1997; Biddinger et al. 1998), no previous data had indicated whether TPSI1 was regulated by non-nutrient abiotic stresses. To address this question, tomato plants were subjected to drought, salt, heat and cold stress, and the expression level of TPSI1 was determined by reverse transcriptionpolymerase chain reaction (RT-PCR) analysis. We first examined the effectiveness of the stress treatments by observing the induced expression levels of Le25, Hsp 70 and $L e C B F 1$ under specific stress conditions (Frank et al. 2009; Kubienová et al. 2013; Li et al. 2013; Zhang et al. 2004). As expected, the expression of Le25 was induced when plants encountered drought (D) and salt (S) stresses, and the expression of Hsp 70 and LeCBF1 was induced in heat- $(\mathrm{H})$ and cold-treated $(\mathrm{C})$ plants, respectively (Fig. S1a). We next examined the Pi concentration in plants under these conditions and found that, except for plants grown under Pi-deficient conditions (-Pi), the remaining stress-treated plants displayed similar or even higher leaf Pi concentrations than those in the non-stress control plants (CK) (Fig. S1b). Consistent with the Pi levels, the expression of TPSI1 in leaves was highly induced only by -Pi treatment and not by drought (D), salt (S), heat (H) or cold (C) stress (Fig. S1c). The above results indicated that TPSI1 is specifically regulated by $\mathrm{Pi}$ and is unaffected by other 
(a)

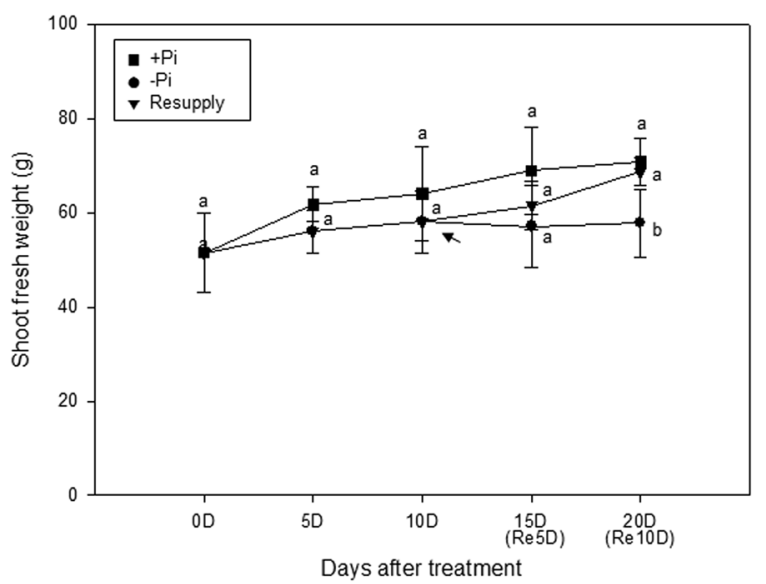

(c)

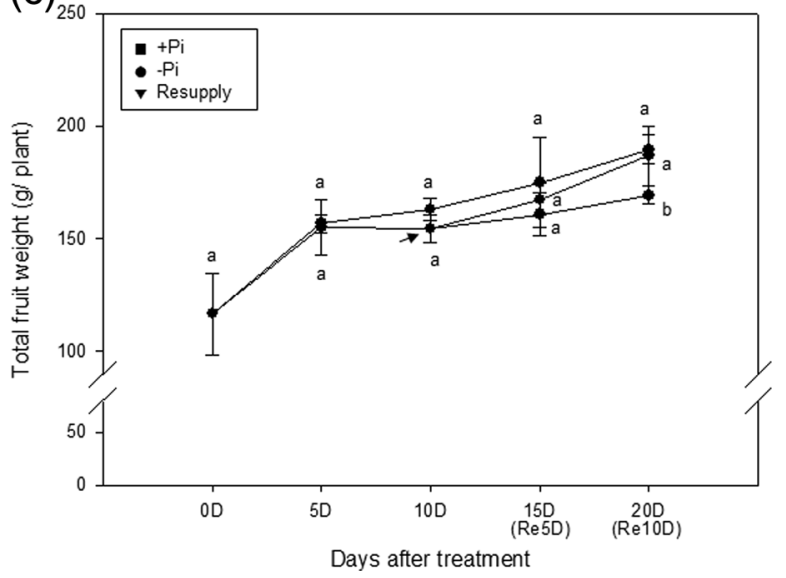

Fig. 5 Effects of Pi treatments for different durations on the yield and quality of tomato during the reproductive stage. Tomato plants were grown under Pi-sufficient $(+\mathrm{Pi})$ conditions or subjected to $\mathrm{Pi}$ deficient (-Pi) and Pi-resupply $(\mathrm{Re})$ treatments for different durations. The shoot fresh weight (a), fruit Pi concentration (b), total fruit weight (c) and total soluble solid content (d) were determined.

nutrient deficiencies or by drought, salt, heat or cold abiotic stresses.

\section{Discussion}

Effects of Pi deficiency on tobacco and tomato growth

During the vegetative stage of tobacco, Pi deficiency significantly reduced the shoot F.W. but not the root F.W. (Fig. 2a and b); accordingly, the calculated root/shoot F.W. (b)

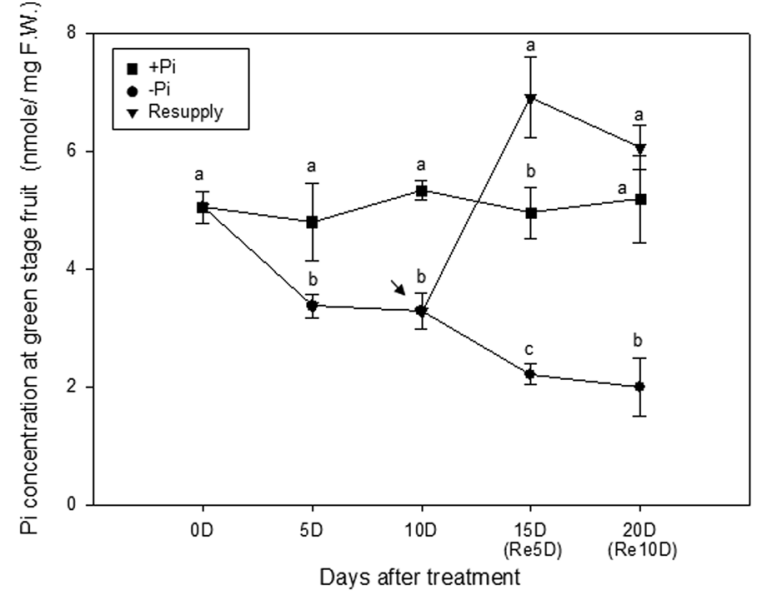

(d)

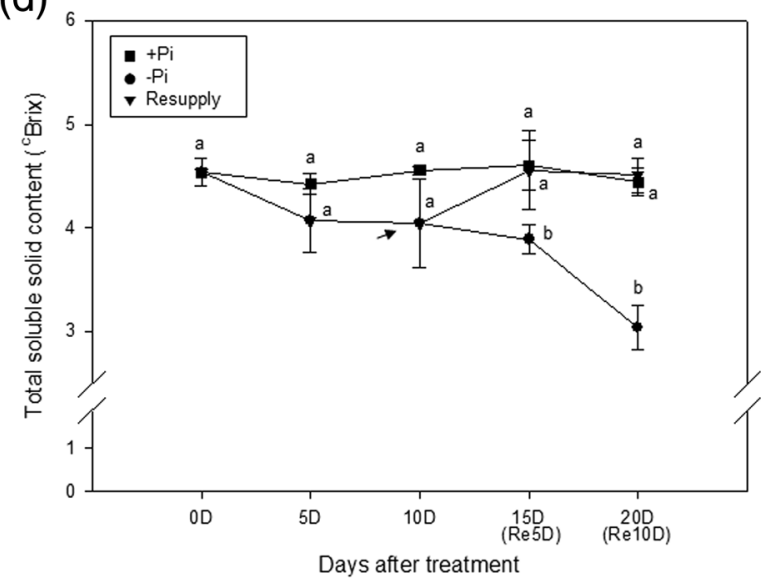

Pi-resupply treatment was initiated after ten days of Pi deficiency (arrow). Different letters at each point denote significant differences between Pi treatments at $p<0.05$ using Fisher's protected LSD test. Error bars represent the SD values of the means of three replicates $(n=3)$ ratio slightly increased (data not shown). During the reproductive stage of tomato, Pi deficiency reduced the shoot F.W. (Fig. 5a) but not the root F.W. (data not shown). These results are similar to those of previous studies in tomato, which showed that Pi starvation reduced the dry weight of roots and shoots and increased the root/shoot biomass ratio (Clarkson and Scattergood 1982; Biddinger et al. 1998). The increased growth of roots is considered as an adaptive response of plants to Pi starvation stress (Lynch 1995; Biddinger et al. 1998; Raghothama 1999). In this study, we further showed that fruit weight and TSS 
(a)

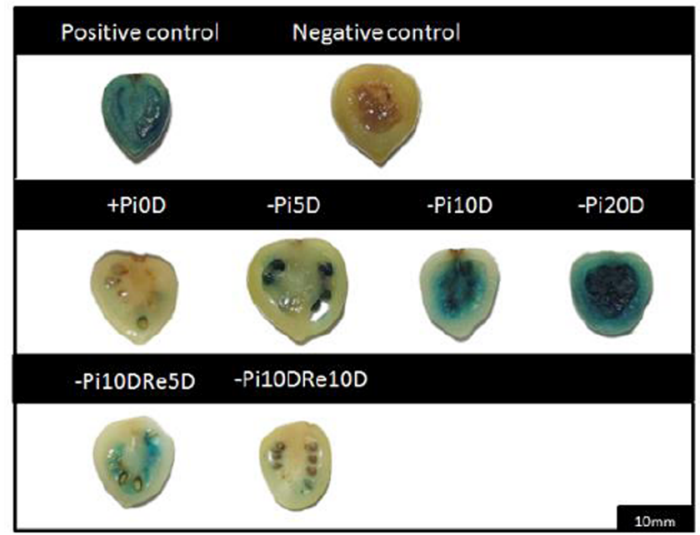

Fig. 6 Effects of Pi treatments for different durations on GUS staining levels and relative GUS activity levels in tomato fruits after three days of agroinjection. a Tomato plants were grown under Pi-sufficient $(+\mathrm{Pi})$ conditions or subjected to Pi-deficient $(-\mathrm{Pi})$ and Pi-resupply (Re) treatments for different durations. Fruits were used for agroinjection and GUS staining. Other than the positive and negative controls, all of the fruits used for GUS staining were transformed with the $\mathrm{P}_{T P S I I}:: G U S$ construct via agroinjection. The positive control was transformed with the $\mathrm{P}_{35 S}: G U S$ construct. The negative control was not transformed with any construct. b Tomato plants were grown under Pisufficient $(+\mathrm{Pi})$ conditions or subjected to Pi-deficient $(-\mathrm{Pi})$ and

content in tomato were also reduced when plants encountered Pi stress (Fig. 5c and 5d). Previous studies showed that Pi starvation reduced the stomatal conductance and photosynthesis rate in tomato (Clarkson and Scattergood 1982; Biddinger et al. 1998; Fujita et al. 2003), possibly explaining why the shoot F.W. and total fruit weight were reduced.

In response to Pi deficiency, the reduction of Pi concentrations is much quicker than that of F.W. or fruit yield and quality. Compared to plants grown under Pi-sufficient conditions, one day of Pi-deficient treatment (-Pi1D) significantly reduced the Pi concentrations in tobacco leaves (Fig. 2c), whereas the reduction of shoot F.W. of tobacco was not observed until 4 days of Pi-deficient treatment (-Pi4D) (Fig. 2a). A similar phenomenon was observed in tomato: 5 days of Pi-deficient treatment (-Pi5D) significantly reduced the Pi concentrations in tomato fruits compared to plants grown under Pi-sufficient conditions (+Pi5D) (Fig. 5b). However, the shoot F.W. (Fig. 5a), the total fruit weight (Fig. 5c) and the TSS content (Fig. 5d) were not significantly reduced until 15-20 days of Pideficient treatment. (b)

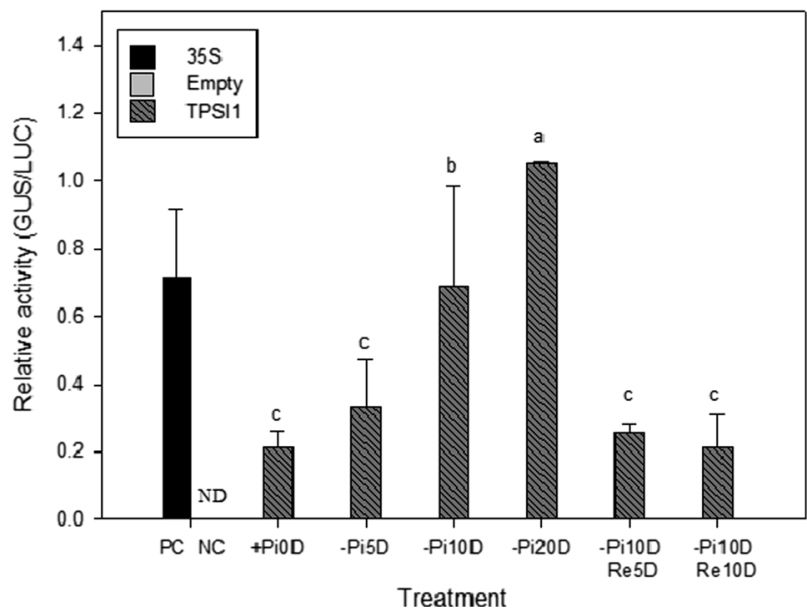

Pi-resupply (Re) treatments for different durations. Fruits were used for agroinjection and GUS activity assays. The fruits used for relative GUS activity assays were transformed with the $\mathrm{P}_{35 S}:$ GUS construct ( $35 \mathrm{~S}$, black bars), the $\mathrm{P}_{\text {TPSII }}:: G U S$ construct (TPSI1, slashed bars) or no construct (Empty, gray bars) via agroinjection. The level of relative GUS activity was normalized to the level of LUC luminescence expressed by the $\mathrm{P}_{355}:$ :LUC construct co-transformed with the $\mathrm{P}_{T P S I 1}:: G U S$ or $\mathrm{P}_{355}:: G U S$ constructs. Means within each panel followed by the same letters are not significantly different between Pi treatments at $p<0.05$ using Fisher's protected LSD test. Error bars represent the SD values of the means of three replicates $(n=3)$

Resupplying Pi to Pi-deficient plants dramatically increased the Pi concentrations in tobacco leaves (Fig. 2c) and roots (Fig. 2d) and in tomato fruits (Fig. 5b). The Pi concentrations at the beginning of the resupply period were even higher than those of plants that were continuously grown under Pi-sufficient conditions. A previous study found that the Pi-absorption rate increased when $\mathrm{Pi}$ was resupplied to Pi-starved tomatoes; this increase continued for several days, resulting in increased $\mathrm{Pi}$ concentrations in shoots and roots (Clarkson and Scattergood 1982). These results were consistent with our findings. One possible explanation for this phenomenon is that several adaptive responses are induced when the plant encounters Pi-deficiency stress, and one of which is the upregulation of high-affinity $\mathrm{Pi}$ transporters to increase Pi uptake (Raghothama 1999; Raghothama and Karthikeyan 2005). When plants were transferred from Pi-deficient conditions to Pisufficient conditions, the expressed and not yet degraded $\mathrm{Pi}$ transporters could help plants take up more Pi. 
The advantages and disadvantages of applying agroinfiltration/agroinjection-based diagnostic systems to monitor $\mathrm{P}$ status in plants

Pi availability is a major factor determining crop productivity (Raghothama and Karthikeyan 2005). Our results showed that -Pi4D treatment significantly reduced tobacco shoot F.W. (Fig. 2a), while Pi deprivation for 1520 days significantly reduced tomato shoot F.W., fruit weight and TSS content (Fig. 5). However, timely resupply of Pi eliminated reductions in final yield and quality. Therefore, the development of a diagnostic system that monitors the Pi status of plants in real-time could provide a guideline for whether plants are in need of fertilization. Our results showed that if Pi stress is identified within 3 days in tobacco or within 10 days in tomato fruit, resupply of Pi fertilizer within these windows will prevent losses in final yield and quality (Figs. 2 and 5). Using the agroinfiltration-based diagnostic system that we developed here, Pi stress could be reflected when the tobacco plant was only Pi-starved for one day (Fig. 3). Additionally, the agroinjection-based diagnostic system was able to reflect Pi stress when the tomato plant was Pistarved for more than five days (Fig. 6). These findings imply that the response of our system was sufficiently rapid, detecting GUS activity before Pi starvation stress start to limit plant growth. The time points at which these agroinfiltration/agroinjection-based diagnostic systems reflect Pi stress (Figs. 3 and 6) are close to when the Pi concentrations changed in tobacco leaves (Fig. 2c) and tomato fruits (Fig. 5b).

Many nutrient deficiencies in soil can affect crop growth. Therefore, the diagnostic systems should be specific. Previous research has shown that the expression of TPSI1 is specifically regulated by $\mathrm{Pi}$ and not by $\mathrm{N}, \mathrm{K}, \mathrm{Fe}, \mathrm{Ca}$ or $\mathrm{Mg}$ (Liu et al. 1997; Biddinger et al. 1998). In this study, the agroinfiltration-based diagnostic system confirmed that TPSII was specifically regulated by Pi and not by N, K, Fe, Ca or Mg (Fig. 4c and d). In addition, we observed that TPSI1 was not regulated by S (Fig. 4c and d). Furthermore, we showed that the expression level of TPSI1 was not changed by drought, salt, heat or cold stress, as determined by RTPCR analysis (Fig. S1c). These results demonstrated that the system is specific for such an application.

In practice, farmers must know whether the application of remedial Pi fertilizer due to $\mathrm{P}$ deficiencies crop is successful. In these agroinfiltration/agroinjection-based diagnostic systems, TPSI1-driven GUS activity was repressed quickly when $\mathrm{Pi}$ was resupplied to $\mathrm{Pi}$ deficient plants (Figs. 3 and 6). This result is consistent with a previous study showing that TPSII mRNA was reduced quickly when $\mathrm{Pi}$ was resupplied to Pi-starved tomato plant (Liu et al. 1997). The reversibility of this system is particularly important because it allows farmers to know whether the use of remedial Pi fertilizer is effective and to avoid over-fertilization.

In the diagnostic system, the purpose of the selected marker is to allow changes in the transcript level of a gene to be observed simply. GUS, green fluorescent protein (GFP), and LUC are commonly used marker genes (de Ruijter et al. 2003). The selected marker should have characteristics that include a short half-life time and easy assay to make the diagnosis procedure rapid and simple (Hammond and White 2008). Hammond et al. selected GUS as the reporter gene for their smart plant (Hammond et al. 2003, 2004; Hammond and White 2008). The advantage of the GUS reporter system is that it can be imaged and quantified in vitro (de Ruijter et al. 2003). Traditional quantification of GUS activity is only performed by in vitro assay, but when it is combined with fluorescent probe, in vivo monitoring of GUS activity becomes possible. The disadvantage of the GUS reporter system is the high intrinsic stability of the GUS protein. Kamiya et al. used LUC as their reporter system (Kamiya et al. 2012). The LUC reporter system is commonly used in mutant screening because it has a low background and a wide dynamic range and can be observed non-invasively (de Ruijter et al. 2003; Kamiya et al. 2012). Regardless of whether a GUS or LUC reporter system is used, specific substrates are required (de Ruijter et al. 2003). In the GFP reporter system, no substrate is needed, and GFP can be visualized in vivo. In the future, the GUS reporter system can be changed to a fluorescent protein (e.g., GFP) for in vivo visualization. However, the autofluorescence from chlorophyll and cell walls in specific tissues often interfere with observation. For both LUC and GFP reporter systems, specific devices are also required for observation. Compared to traditional chemical analyses that use ICP spectroscopic techniques to determine $\mathrm{P}$ contents in plant materials, the agroinfiltration/agroinjection-based diagnostic systems do not require expensive equipment and complicated techniques, making diagnosis more convenient and economical. GUS-staining analysis is relatively easy but only qualitative, while the quantitative analysis of GUS activity requires additional equipment. 
The traditional smart plant system requires a long period to obtain transgenic plants and has only been used in crops for which transformation and regeneration procedures have been developed. Unlike the traditional smart plant system, the agroinfiltration/agroinjection-based diagnostic systems do not require the generation of stable transgenic plants, broadening their applications. In the future, the sensitivity of the developed system should be tested using different Pi fertilizer application regimes. Furthermore, the index plants used for agroinfiltration/ agroinjection need to be carefully monitored when $A$. tumefaciens is applied on-site in the field because of the concern of genetically modified crops. Alternatively, transient expression of a $\mathrm{P}_{T P S I I}:: G U S$ construct could be applied in detached leaves or fruits via vacuum infiltration in the lab, but the variable results and weak expression level must be overcome (Kapila et al. 1997; Fu et al. 2005; Wroblewski et al. 2005; Orzaez et al. 2006). In addition, diagnostic microarrays examining the global gene expression profile have been proposed for the diagnosis of Pi status in plants (Hammond et al. 2011); however, microarray analyses remains very expensive. Very recently, a method that monitors changes in the shape of time-dependent transient fluorescence using a chlorophyll fluorimeter was developed to diagnose $\mathrm{P}$ status in plants (Frydenvang et al. 2015), providing an alternative method for monitoring $\mathrm{P}$ status in plants.

Potential applications of agroinfiltration/ agroinjection-based diagnostic systems

The excessive application of Pi fertilizer is common in developed countries and may destroy the ecosystem. Farmers in undeveloped countries may not be able to afford expensive Pi fertilizer, and their crops may suffer from Pi deficiency. The agroinfiltration/agroinjectionbased diagnostic systems developed in this study can be used in both leaves and fruits, and they are sufficiently rapid and specific to the $\mathrm{Pi}$ stress response. Furthermore, the systems are reversible, convenient and economical when combined with GUS staining, making optimal fertilization possible and easy. In practice, plants can be randomly selected from the field for agroinfiltration/agroinjection. The farmer can be advised where and whether they need to apply fertilizer. In total, 8-16 individual transient expression assays have been demonstrated in a single tobacco leaf (Yang et al. 2000). Therefore, this technique has the potential for use in the diagnosis of diverse nutrient statuses in crops through the simultaneous combination of different promoters and reporter genes.

In our results, we provide a proof-of-concept study for $\mathrm{Pi}$ status monitoring via the response of a $\mathrm{Pi}$ starvation-induced gene that can be introduced by agroinfiltration/agroinjection into tobacco and tomato. In addition to tobacco, agroinfiltration has been successfully applied to many plant species such as Arabidopsis and lettuce (Lactuca sativa) (Wroblewski et al. 2005). Using the $\mathrm{P}_{35 S}:$ :GUS construct, we also found that agroinfiltration can successfully work in eggplant (data not shown). Furthermore, agroinjection was successful in tomato fruit and has been applied in apple (Malus $\times$ domestica), pear (Pyrus communis), peach (Prunus persica), strawberry (Fragaria $\times$ ananassa) and orange (Citrus sinensis) (Spolaore et al. 2001). Therefore, the systems described in this study also have potential applications in other crops.

\section{Conclusions}

To apply the concept of smart plants (Hammond et al. 2003) to crops, we developed agroinfiltration/ agroinjection-based diagnostic systems that allow for the precise management of Pi fertilization and that can be easily used for crops such as tomato. Furthermore, this system is sufficiently specific and rapid to reflect the $\mathrm{Pi}$ status in plants before $\mathrm{P}$ starvation stress reduces plant growth and crop yield, providing farmers with adequate time to apply Pi fertilizer. In addition, reductions in GUS activity in plants resupplied with Pi can help farmers avoid under- or over-fertilization, which is important for sustainable agriculture. These methods are simple, convenient, and economical and do not require expensive equipment. Our system provides a guideline for fertilization with $\mathrm{Pi}$ when necessary without compromising crop yield or quality.

Acknowledgments This research was supported by grants from the National Science Council (100-2313-B-002-058-MY2) and the Ministry of Science and Technology (102-2313-B-002-012MY3) of the Republic of China to S.-I.L. Experiments and data analysis were performed in part through the use of the inductively coupled plasma optical emission spectrometer (ICP-OES) at the Agricultural Biotechnology Research Center of Academia Sinica, with the assistance of Yan-Chen Huang. We thank Yao-Chien Alex Chang and Jen-An Lin in the Department of Horticulture and Landscape Architecture, National Taiwan University, for assistance with the total $\mathrm{N}$ analysis. 


\section{References}

Adesanwo OO, Ige DV, Thibault L, Flaten D, Akinremi W (2013) Comparison of colorimetric and ICP methods of phosphorus determination in soil extracts. Commun Soil Sci Plant Anal 44:3061-3075. doi:10.1080/00103624.2013.832771

Ames BN (1966) Assay of inorganic phosphate, total phosphate and phosphatases. Methods Enzymol 8:115-118

Bendahmane A, Querci M, Kanyuka K, Baulcombe DC (2000) Agrobacterium transient expression system as a tool for the isolation of disease resistance genes: application to the $R \times 2$ locus in potato. Plant J 21:73-81. doi:10.1046/j.1365-313x. 2000.00654.x

Biddinger EJ, Liu C, Joly RJ, Raghothama KG (1998) Physiological and molecular responses of aeroponically grown tomato plants to phosphorus deficiency. J Am Soc Hortic Sci 123:330-333

Bradford MM (1976) A rapid and sensitive method for the quantitation of microgram quantities of protein utilizing the principle of protein-dye binding. Anal Biochem 72:248-254. doi: 10.1016/0003-2697(76)90527-3

Burleigh SH, Harrison MJ (1997) A novel gene whose expression in Medicago truncatula roots is suppressed in response to colonization by vesicular-arbuscular mycorrhizal (VAM) fungi and to phosphate nutrition. Plant Mol Biol 34:199208. doi:10.1023/A:1005841119665

Burleigh SM, Harrison MJ (1998) Characterization of the Mt4 gene from Medicago truncatula. Gene 216:47-53. doi:10. 1016/S0378-1119(98)00326-6

Burleigh SH, Harrison MJ (1999) The down-regulation of Mt4like genes by phosphate fertilization occurs systemically and involves phosphate translocation to the shoots. Plant Physiol 119:241-248. doi:10.1104/pp.119.1.241

Chiou T-J, Lin S-I (2011) Signaling network in sensing phosphate availability in plants. Annu Rev Plant Biol 62:185-206. doi: 10.1146/annurev-arplant-042110-103849

Chiou T-J, Aung K, Lin S-I, Wu C-C, Chiang S-F, C-1 S (2006) Regulation of phosphate homeostasis by microRNA in Arabidopsis. Plant Cell 18:412-421. doi:10.1105/tpc.105. 038943

Clarkson DT, Scattergood CB (1982) Growth and phosphate transport in barley and tomato plants during the development of, and recovery from, phosphate-stress. J Exp Bot 33:865875. doi: $10.1093 / \mathrm{jxb} / 33.5 .865$

Cordell D, White S (2014) Life's bottleneck: sustaining the world's phosphorus for a food secure future. Annu Rev Environ Resour 39:161-188. doi:10.1146/annurev-environ-010213113300

Cordell D, Drangert J-O, White S (2009) The story of phosphorus: global food security and food for thought. Glob Environ Chang 19:292-305. doi:10.1016/j.gloenvcha.2008.10.009

Curtis MD, Grossniklaus U (2003) A gateway cloning vector set for high-throughput functional analysis of genes in planta. Plant Physiol 133:462-469. doi:10.1104/pp.103.027979

de Ruijter NCA, Verhees J, van Leeuwen W, van der Krol AR (2003) Evaluation and comparison of the GUS, LUC and GFP reporter system for gene expression studies in plants. Plant Biol 5:103-115. doi:10.1055/s-2003-40722

Essigmann B, Güler S, Narang RA, Linke D, Benning C (1998) Phosphate availability affects the thylakoid lipid composition and the expression of $S Q D 1$, a gene required for sulfolipid biosynthesis in Arabidopsis thaliana. Proc Natl Acad Sci U S A 95:1950-1955. doi:10.1073/pnas.95.4.1950

Frank G, Pressman E, Ophir R, Althan L, Shaked R, Freedman M, Shen S, Firon N (2009) Transcriptional profiling of maturing tomato (Solanum lycopersicum L.) microspores reveals the involvement of heat shock proteins, ROS scavengers, hormones, and sugars in the heat stress response. J Exp Bot 60: 3891-3908. doi:10.1093/jxb/erp234

Frydenvang J, van Maarschalkerweerd M, Carstensen A, Mundus S, Schmidt SB, Pedas PR, Laursen KH, Schjoerring JK, Husted S (2015) Sensitive detection of phosphorus deficiency in plants using chlorophyll a fluorescence. Plant Physiol 169:353-361. doi:10.1104/pp.15.00823

Fu D-Q, Zhu B-Z, Zhu H-L, Jiang W-B, Luo Y-B (2005) Virusinduced gene silencing in tomato fruit. Plant J 43:299-308. doi:10.1111/j.1365-313X.2005.02441.X

Fujita K, Okada M, Lei K, Ito J, Ohkura K, Adu-Gyamfi JJ, Mohapatra PK (2003) Effect of P-deficiency on photoassimilate partitioning and rhythmic changes in fruit and stem diameter of tomato (Lycopersicon esculentum) during fruit growth. J Exp Bot 54:2519-2528. doi:10.1093/ jxb/erg 273

Gaxiola RA, Edwards M, Elser JJ (2011) A transgenic approach to enhance phosphorus use efficiency in crops as part of a comprehensive strategy for sustainable agriculture. Chemosphere 84:840-845. doi:10.1016/j.chemosphere. 2011.01.062

Gilbert N (2009) Environment: the disappearing nutrient. Nature 461:716-718. doi:10.1038/461716a

Hammond JP, White PJ (2008) Diagnosing phosphorus deficiency in crop plants. In: PJ W, JP H (eds) The ecophysiology of plant-phosphorus interactions, vol. 7. Plant ecophysiology. Springer-Verlag, Dordrecht, the Netherlands, pp. 225-246

Hammond JP, Bennett MJ, Bowen HC, Broadley MR, Eastwood DC, May ST, Rahn C, Swarup R, Woolaway KE, White PJ (2003) Changes in gene expression in Arabidopsis shoots during phosphate starvation and the potential for developing smart plants. Plant Physiol 132:578-596. doi:10.1104/pp. 103.020941

Hammond JP, White PJ, Broadley MR (2004) Diagnosing phosphorus deficiency in plants. Asp. Appl Bioinforma 72:89-98

Hammond JP, Broadley MR, Bowen HC, Spracklen WP, Hayden RM, White PJ (2011) Gene expression changes in phosphorus deficient potato (Solanum tuberosum L.) leaves and the potential for diagnostic gene expression markers. PLoS One 6:e24606. doi:10.1371/journal.pone.0024606

Hoagland DR, Arnon DI (1950) The water-culture method for growing plants without soil. University of California, Berkeley, CA, College of Agriculture

Hofgen R, Willmitzer L (1988) Storage of competent cells for agrobacterium transformation. Nucleic Acids Res 16:9877. doi:10.1093/nar/16.20.9877

Holford ICR (1997) Soil phosphorus: its measurement, and its uptake by plants. Aust J Soil Res 35:227-239. doi:10.1071/ S96047

Hou XL, Wu P, Jiao FC, Jia QJ, Chen HM, Yu J, Song XW, Yi KK (2005) Regulation of the expression of OSIPS1 and OsIPS2 in rice via systemic and local pi signalling and hormones. Plant Cell Environ 28:353-364. doi:10.1111/j.1365-3040. 2005.01272.x 
Jefferson RA, Kavanagh TA, Bevan MW (1987) GUS fusions: beta-glucuronidase as a sensitive and versatile gene fusion marker in higher plants. EMBO J 6:3901-3907

Kamiya T, Yamagami M, Hirai MY, Fujiwara T (2012) Establishment of an in planta magnesium monitoring system using CAX3 promoter-luciferase in Arabidopsis. J Exp Bot 63:355-363. doi:10.1093/jxb/err283

Kapila J, De Rycke R, Van Montagu M, Angenon G (1997) An Agrobacterium-mediated transient gene expression system for intact leaves. Plant Sci 122:101-108. doi:10.1016/ S0168-9452(96)04541-4

Kubienová L, Sedlářová M, Vítečková-Wünschová A, Piterková J, Luhová L, Mieslerová B, Lebeda A, Navrátil M, Petrrivalský M (2013) Effect of extreme temperatures on powdery mildew development and Hsp70 induction in tomato and wild Solanum spp. Plant Prot Sci 49:S41-S54

Li Z, Zhang L, Wang A, Xu X, Li J (2013) Ectopic overexpression of SlHsfA3, a heat stress transcription factor from tomato, confers increased thermotolerance and salt hypersensitivity in germination in transgenic Arabidopsis. PLoS One 8: e54880. doi:10.1371/journal.pone.0054880

Lin W-Y, Lin S-I, Chiou T-J (2009) Molecular regulators of phosphate homeostasis in plants. J Exp Bot 60:1427-1438. doi:10.1093/jxb/ern303

Liu C, Muchhal US, Raghothama KG (1997) Differential expression of TPS11, a phosphate starvation-induced gene in tomato. Plant Mol Biol 33:867-874. doi:10.1023/ A:1005729309569

Liu L, Zhang Y, Tang S, Zhao Q, Zhang Z, Zhang H, Dong L, Guo H, Xie Q (2010) An efficient system to detect protein ubiquitination by agroinfiltration in nicotiana benthamiana. Plant J 61:893-903. doi:10.1111/j.1365-313X.2009.04109.x

Liu T-Y, Huang T-K, Tseng C-Y, Lai Y-S, Lin S-I, Lin W-Y, Chen J-W, Chiou T-J (2012) PHO2-dependent degradation of PHO1 modulates phosphate homeostasis in Arabidopsis. Plant Cell 24:2168-2183. doi:10.1105/tpc.112.096636

Lynch J (1995) Root architecture and plant productivity. Plant Physiol 109:7-13

Martín AC, del Pozo JC, Iglesias J, Rubio V, Solano R, de la Peña A, Leyva A, Paz-Ares J (2000) Influence of cytokinins on the expression of phosphate starvation responsive genes in Arabidopsis. Plant J 24:559-567. doi:10.1046/j.1365-313x. 2000.00893.x

Millner PD, Kitt DG (1992) The Beltsville method for soilless production of vesicular-arbuscular mycorrhizal fungi. Mycorrhiza 2:9-15. doi:10.1007/BF00206278

Murphy J, Riley JP (1962) A modified single solution method for the determination of phosphate in natural waters. Anal Chim Acta 27:31-36. doi:10.1016/S0003-2670(00)88444-5

Orzaez D, Mirabel S, Wieland WH, Granell A (2006) Agroinjection of tomato fruits. A tool for rapid functional analysis of transgenes directly in fruit. Plant Physiol 140:311. doi:10.1104/pp.105.068221

Pittman JJ, Zhang H, Schroder JL, Payton ME (2005) Differences of phosphorus in Mehlich 3 extracts determined by colorimetric and spectroscopic methods. Commun Soil Sci Plant Anal 36:1641-1659. doi:10.1081/CSS-200059112

Raghothama KG (1999) Phosphate acquisition. Annu Rev Plant Physiol Plant Mol Biol 50:665-693. doi:10.1146/annurev. arplant.50.1.665

Raghothama KG, Karthikeyan AS (2005) Phosphate acquisition. Plant Soil 274:37-49. doi:10.1007/s11104-004-2005-6

Schachtman DP, Reid RJ, Ayling SM (1998) Phosphorus uptake by plants: from soil to cell. Plant Physiol 116:447-453. doi: 10.1104/pp.116.2.447

Schöb H, Kunz C, Meins F Jr (1997) Silencing of transgenes introduced into leaves by agroinfiltration: a simple, rapid method for investigating sequence requirements for gene silencing. Mol Gen Genet 256:581-585. doi:10.1007/ s004380050604

Shin H, Shin H-S, Chen R, Harrison MJ (2006) Loss of At4 function impacts phosphate distribution between the roots and the shoots during phosphate starvation. Plant J 45:712726. doi:10.1111/j.1365-313X.2005.02629.x

Spolaore S, Trainotti L, Casadoro G (2001) A simple protocol for transient gene expression in ripe fleshy fruit mediated by agrobacterium. J Exp Bot 52:845-850

Vance CP, Uhde-Stone C, Allan DL (2003) Phosphorus acquisition and use: critical adaptations by plants for securing a nonrenewable resource. New Phytol 157:423-447. doi:10. 1046/j.1469-8137.2003.00695.x

Wasaki J, Yonetani R, Shinano T, Kai M, Osaki M (2003) Expression of the OsPII gene, cloned from rice roots using cDNA microarray, rapidly responds to phosphorus status. New Phytol 158:239-248. doi:10.1046/j.1469-8137.2003. 00748.x

Withers PJA, Edwards AC, Foy RH (2001) Phosphorus cycling in UK agriculture and implications for phosphorus loss from soil. Soil Use Manag 17:139-149. doi:10.1079/SUM200181

Wroblewski T, Tomczak A, Michelmore R (2005) Optimization of Agrobacterium-mediated transient assays of gene expression in lettuce, tomato and Arabidopsis. Plant Biotechnol J 3:259273. doi:10.1111/j.1467-7652.2005.00123.x

Yang Y, Li R, Qi M (2000) In vivo analysis of plant promoters and transcription factors by agroinfiltration of tobacco leaves. Plant J 22:543-551. doi:10.1046/j.1365-313x.2000.00760.x

Zhang X, Fowler SG, Cheng H, Lou Y, Rhee SY, Stockinger EJ, Thomashow MF (2004) Freezing-sensitive tomato has a functional $\mathrm{CBF}$ cold response pathway, but a $\mathrm{CBF}$ regulon that differs from that of freezing-tolerant Arabidopsis. Plant $\mathrm{J}$ 39:905-919. doi:10.1111/j.1365-313X.2004.02176.x 